\title{
Reply to Letter to the Editor
}

\section{Early Outcome of TKA with a Medial Pivot Fixed-bearing Prosthesis Is Worse Than With a PFC Mobile-bearing Prosthesis}

\author{
Young-Hoo Kim MD, Sung-Hwan Yoon MD, \\ Jun-Shik Kim MD
}

Published online: 25 October 2008

(C) The Association of Bone and Joint Surgeons 2008

We appreciate Dr. Pritchett's comments regarding our article, "Early Outcome of TKA with a Medial Pivot Fixed-bearing Prosthesis is Worse Than With a PFC Mobile-bearing Prosthesis" [3].

We congratulate Dr. Pritchett on his ability to obtain good results with the Medial Pivot TKA. We agree with him completely regarding resection of the posterior cruciate ligament (PCL) and resection of more of the medial femoral condyle when TKA is performed with a Medial Pivot prosthesis. We believe the $16 \%$ of the Medial Pivot TKAs in our series that had recession of the PCL had higher functional scores compared with TKAs without having recession of the PCL.

In our previous studies [1, 2], we achieved high functional scores with mobile- and fixed-bearing prostheses of similar or different design at midterm followup. Therefore, we believe the lower scores with a Medial Pivot TKA in our series [3] are attributable to surgical technique and are design specific.
We recommend complete resection of the PCL and resection of more of the medial femoral condyle when performing the TKA with a Medial Pivot knee prosthesis.

\section{References}

1. Kim YH, Kim DY, Kim JS. Simultaneous mobile- and fixedbearing total knee replacement in the same patients: a prospective comparison of mid-term outcomes using a similar design of prosthesis. J Bone Joint Surg Br. 2007;89:904-910.

2. Kim YH, Kook HK, Kim JS. Comparison of fixed-bearing and mobile-bearing total knee arthroplasties. Clin Orthop Relat Res. 2001;392:101-115.

3. Kim YH, Yoon SH, Kim JS. Early outcome of TKA with a Medial Pivot fixed-bearing prosthesis is worse than with a PFC mobilebearing prosthesis. Clin Orthop Relat Res. 2008 May 9. [Epub ahead of print].
Y.-H. Kim ( $ه)$, S.-H. Yoon, J.-S. Kim

The Joint Replacement Center of Korea, Ewha Womans

University School of Medicine, 911-1, MokDong,

YangCheon-Ku, Seoul 158-710, South Korea

e-mail: younghookim@ewha.ac.kr 